\title{
THE DEGREE OF THE ALGEBRA OF COVARIANTS OF A BINARY FORM
}

\author{
LEONID BEDRATYUK AND NADIA ILASH
}

ABSTRACT. We calculate the degree of the algebra of covariants $\mathcal{C}_{d}$ for binary $d$-forms. We obtain the integral representation and asymptotic behavior of the degree.

1. Introduction. Let $R=R_{0} \oplus R_{1} \oplus \cdots, R_{0}=\mathbb{C}$, be a finitely generated graded commutative $\mathbb{C}$-algebra without zero divisors. Denote by

$$
\mathcal{P}(R, z)=\sum_{j=0}^{\infty} \operatorname{dim} R_{j} z^{j},
$$

its Poincaré series. Letting $r$ be the transcendence degree of the quotient field of $R$ over $\mathbb{C}$, the number

$$
\operatorname{deg}(R):=\lim _{z \rightarrow 1}(1-z)^{r} \mathcal{P}(R, z),
$$

is called the degree of the algebra $R$. The first two terms of the Laurent series expansion of $\mathcal{P}(R, z)$ at the point $z=1$ have the following form

$$
\mathcal{P}(R, z)=\frac{\operatorname{deg}(R)}{(1-z)^{r}}+\frac{\psi(R)}{(1-z)^{r-1}}+\cdots .
$$

The numbers $\operatorname{deg}(R)$ and $\psi(R)$ are important characteristics of the algebra $R$. For instance, if $R$ is an algebra of invariants of a finite group $G$, then $\operatorname{deg}(R)^{-1}$ is an order of the group $G$ and $2 \frac{\psi(R)}{\operatorname{deg}(R)}$ is the number of pseudo-reflections in $G$, see [2].

Let $V_{d}$ be the standard $(d+1)$-dimensional complex representation of $S L_{2}$, and let $\mathcal{I}_{d}:=\mathbb{C}\left[V_{d}\right]^{S L_{2}}$ be the corresponding algebra of invariants. In the language of classical invariant theory the algebra $\mathcal{I}_{d}$ is called the

2010 AMS Mathematics subject classification. Primary 13A50, 13N15.

Keywords and phrases. Classical invariant theory, algebra of invariants of binary form, algebra of covariants of binary form, Poincaré series, degree of algebra.

Received by the editors on December 27, 2013, and in revised form on July 2, 2014. 
algebra of invariants for binary forms of degree $d$. The following explicit formula for the degree $\operatorname{deg}\left(\mathcal{I}_{d}\right)$ was derived by Hilbert in [4]:

$$
\operatorname{deg}\left(\mathcal{I}_{d}\right)=\left\{\begin{array}{l}
-\frac{1}{4 d !} \sum_{0 \leq e<d / 2}(-1)^{e}\left(\begin{array}{l}
d \\
e
\end{array}\right)\left(\frac{d}{2}-e\right)^{d-3}, \text { if } d \text { is odd } \\
-\frac{1}{2 d !} \sum_{0 \leq e<d / 2}(-1)^{e}\left(\begin{array}{l}
d \\
e
\end{array}\right)\left(\frac{d}{2}-e\right)^{d-3}, \text { if } d \text { is even }
\end{array}\right.
$$

In $[6,7]$, Springer obtained two different proofs of this result. Also, he found an integral representation and the asymptotic behavior for Hilbert's constants. For this purpose, Springer [7] derived an explicit formula for the Poincaré series $\mathcal{P}\left(\mathcal{I}_{d}, z\right)$.

Let $\mathcal{C}_{d}$ be the algebra of the covariants of binary $d$-forms, i.e., $\mathcal{C}_{d} \cong \mathbb{C}\left[V_{1} \oplus V_{d}\right]^{S L_{2}}$. In the present paper, acting in the spirit of Springer's papers, we calculate $\operatorname{deg}\left(\mathcal{C}_{d}\right)$ and $\psi\left(\mathcal{C}_{d}\right)$. The following formulas hold:

$$
\operatorname{deg}\left(\mathcal{C}_{d}\right)=\frac{1}{d !} \sum_{0 \leq j<d / 2}(-1)^{j}\left(\begin{array}{l}
d \\
j
\end{array}\right)\left(\frac{d}{2}-j\right)^{d-1}
$$

and

$$
\psi\left(\mathcal{C}_{d}\right)=\frac{1}{2} \operatorname{deg}\left(\mathcal{C}_{d}\right)
$$

Also, we calculate both an integral representation and the asymptotic behavior of the constants. For this purpose we use the explicit formula for the Poincaré series $\mathcal{P}\left(\mathcal{C}_{d}, z\right)$ derived by the first author in [1].

2. Computation of $\operatorname{deg}\left(\mathcal{C}_{d}\right)$. The algebra of covariants $\mathcal{C}_{d}$ is a finitely generated graded algebra

$$
\mathcal{C}_{d}=\left(\mathcal{C}_{d}\right)_{0} \oplus\left(\mathcal{C}_{d}\right)_{1} \oplus \cdots \oplus\left(\mathcal{C}_{d}\right)_{i} \oplus \cdots,
$$

where the subspaces $\left(\mathcal{C}_{d}\right)_{i}$ of covariants of degree $i$ are each finitedimensional, and $\left(\mathcal{C}_{d}\right)_{0} \cong \mathbb{C}$. The formal power series

$$
\mathcal{P}\left(\mathcal{C}_{d}, z\right)=\sum_{i=0}^{\infty} \operatorname{dim}\left(\left(\mathcal{C}_{d}\right)_{i}\right) z^{i},
$$


is called the Poincaré series of the algebra of covariants $\mathcal{C}_{d}$. The finite generation of $\mathcal{C}_{d}$ implies that its Poincaré series is the power series expansion of a rational function.

The following theorem shows an explicit form for this rational function. Let $\varphi_{n}, n \in \mathbb{N}$, be the linear operator that transforms a rational function $f$ in $z$ to a rational function $\varphi_{n}(f)$ which is defined on the power $z^{n}$ by

$$
\left(\varphi_{n}(f)\right)\left(z^{n}\right)=\frac{1}{n} \sum_{j=0}^{n-1} f\left(\zeta_{n}^{j} z\right), \quad \zeta_{n}=e^{2 \pi i / n} .
$$

Theorem 2.1 ([1]). The Poincaré series $\mathcal{P}\left(\mathcal{C}_{d}, z\right)$ has the following form:

$$
\mathcal{P}\left(\mathcal{C}_{d}, z\right)=\sum_{0 \leq j<d / 2} \varphi_{d-2 j}\left(\frac{(-1)^{j} z^{j(j+1)}(1+z)}{\left(z^{2}, z^{2}\right)_{j}\left(z^{2}, z^{2}\right)_{d-j}}\right)
$$

where $(a, q)_{n}=(1-a)(1-a q) \cdots\left(1-a q^{n-1}\right)$ denotes the $q$-shifted factorial.

It is well known that the transcendence degree of the quotient field for the algebra of covariants $\mathcal{C}_{d}$ over $\mathbb{C}$ coincides with the order of pole $z=1$ for the rational function $\mathcal{P}\left(\mathcal{C}_{d}, z\right)$ and equals $d$. Therefore, the first terms of the Laurent series for $\mathcal{P}\left(\mathcal{C}_{d}, z\right)$ at the point $z=1$ are

$$
\mathcal{P}\left(\mathcal{C}_{d}, z\right)=\frac{\operatorname{deg}\left(\mathcal{C}_{d}\right)}{(1-z)^{d}}+\frac{\psi\left(\mathcal{C}_{d}\right)}{(1-z)^{d-1}}+\cdots .
$$

In order to calculate the rational coefficients $\operatorname{deg}\left(\mathcal{C}_{d}\right)$ and $\psi\left(\mathcal{C}_{d}\right)$ we shall prove several auxiliary facts.

Lemma 2.2. The following statements hold:

(i) the first terms of the Taylor series for the function $\left(z^{2}, z^{2}\right)_{j}$ at $z=1$ are $\left(z^{2}, z^{2}\right)_{j}=2^{j} j !(1-z)^{j}-2^{j-1} j ! j^{2}(1-z)^{j+1}+\cdots ;$

(ii) the first terms of the Laurent series for the function

$$
\frac{(-1)^{j} z^{j(j+1)}(1+z)}{\left(z^{2}, z^{2}\right)_{j}\left(z^{2}, z^{2}\right)_{d-j}} \quad \text { at } z=1
$$


are:

$$
\frac{(-1)^{j}}{2^{d-1} j !(d-j) !} \frac{1}{(1-z)^{d}}+\frac{(-1)^{j}(d+1)}{2^{d} j !(d-j) !}(d-2 j-1) \frac{1}{(1-z)^{d-1}}+\cdots .
$$

Proof.

(i) We have

$$
\left(z^{2}, z^{2}\right)_{j}=\left(1-z^{2}\right)\left(1-z^{4}\right) \cdots\left(1-z^{2 j}\right) .
$$

Let us expand the polynomial $1-z^{n}$ in a Taylor series about $z=1$. We have

$$
\begin{aligned}
1-z^{n} & =-n(z-1)-\frac{n(n-1)}{2 !}(z-1)^{2}+\cdots \\
& =n(1-z)-\frac{n(n-1)}{2 !}(1-z)^{2}+O\left((1-z)^{3}\right) .
\end{aligned}
$$

Therefore,

$$
\begin{aligned}
\left(z^{2}, z^{2}\right)_{j} & =\left(1-z^{2}\right)\left(1-z^{4}\right) \cdots\left(1-z^{2 j}\right) \\
& =\left(2(1-z)-\frac{2}{2 !}(1-z)^{2}+\cdots\right) \\
& \times\left(4(1-z)-\frac{4 \cdot 3}{2 !}(1-z)^{2}+\cdots\right) \\
& \times\left(2 j(1-z)-\frac{2 j(2 j-1)}{2 !}(1-z)^{2}+\cdots\right) \\
& =\left(2 \cdot 4 \cdots 2 j(1-z)^{j}+(1+3+5 \cdots+2 j-1)\right. \\
& \left.\times 2^{j-1} j !(1-z)^{j+1}+\cdots\right) \\
& =2^{j} j !(1-z)^{j}-2^{j-1} j ! j^{2}(1-z)^{j+1}+\cdots .
\end{aligned}
$$

It follows that

$$
\begin{aligned}
\left(z^{2}, z^{2}\right)_{j}\left(z^{2}, z^{2}\right)_{d-j} & =\left(2^{j} j !(1-z)^{j}-2^{j-1} j ! j^{2}(1-z)^{j+1}+\cdots\right) \\
& \times\left(2^{d-j}(d-j) !(1-z)^{d-j}-2^{d-j-1}(d-j) !\right. \\
& \left.\times(d-j)^{2}(1-z)^{d-j+1}+\cdots\right) \\
& =2^{d} j !(d-j) !(1-z)^{d}-2^{d-1} j !(d-j) ! \\
& \times\left((d-j)^{2}+j^{2}\right)(1-z)^{d+1}+\cdots
\end{aligned}
$$


(ii) To find the first terms of the Laurent series for the function

$$
\frac{(-1)^{j} z^{j(j+1)}(1+z)}{\left(z^{2}, z^{2}\right)_{j}\left(z^{2}, z^{2}\right)_{d-j}}
$$

we expand the numerator in the Taylor series expressed in terms of powers of $(1-z)$. We have:

$$
\begin{aligned}
1+z & =2-(1-z), \\
z^{j(j+1)} & =1-j(j+1)(1-z)+\cdots, \\
(1+z) z^{j(j+1)} & =2-(2 j(j+1)+1)(1-z)+\cdots .
\end{aligned}
$$

It is easy to check that the following decomposition holds:

$$
\frac{a_{0}+a_{1} x+\cdots}{b_{0}+b_{1} x+\cdots}=\frac{a_{0}}{b_{0}}+\frac{a_{1} b_{0}-a_{0} b_{1}}{b_{0}^{2}} x+\cdots, b_{0} \neq 0 .
$$

Then

$$
\begin{aligned}
& \frac{(-1)^{j} z^{j(j+1)}(1+z)}{\left(z^{2}, z^{2}\right)_{j}\left(z^{2}, z^{2}\right)_{d-j}} \\
& =\frac{2-(2 j(j+1)+1)(1-z)+\cdots}{2^{d} j !(d-j) !(1-z)^{d}-2^{d-1} j !(d-j) !\left((d-j)^{2}+j^{2}\right)(1-z)^{d+1}+\cdots} \\
& =\frac{1}{(1-z)^{d}} \frac{2-(2 j(j+1)+1)(1-z)+\cdots}{2^{d} j !(d-j) !-2^{d-1} j !(d-j) !\left((d-j)^{2}+j^{2}\right)(1-z)+\cdots} \\
& =\frac{1}{(1-z)^{d}}\left(\frac{1}{2^{d-1} j !(d-j) !}+\frac{(-1)^{j}(d+1)}{2^{d} j !(d-j) !}(d-2 j-1)(1-z)+\cdots\right)
\end{aligned}
$$

The following lemma shows how the function $\varphi_{n}$ acts on the negative powers of $1-z$.

Lemma 2.3. For $h \in \mathbb{N}$,

$$
\varphi_{n}\left(\frac{1}{(1-z)^{h}}\right)=\sum_{i=0}^{h} \frac{\alpha_{n i}}{(1-z)^{i}}
$$

where $\alpha_{n h}=n^{h-1}$ and $\alpha_{n, h-1}=-n^{h-2}(n-1) h / 2$. 
Proof. Using article [1, Lemma 4], we get

$$
\varphi_{n}\left(\frac{1}{(1-z)^{h}}\right)=\frac{\varphi_{n}\left(\left(1+z+z^{2}+\cdots+z^{n-1}\right)^{h}\right)}{(1-z)^{h}}
$$

Obviously, $\alpha_{n h}$ is the remainder after the division of $\varphi_{n}\left(\left(1+z+z^{2}+\right.\right.$ $\left.\left.\cdots+z^{n-1}\right)^{h}\right)$ by $(1-z)$.

Using the definition of the function $\varphi_{n}$ we get

$$
\begin{aligned}
\varphi_{n}\left(\left(1+z+z^{2}+\cdots+z^{n-1}\right)^{h}\right) & \\
& =\left.\frac{1}{n} \sum_{j=0}^{n-1}\left(1+\zeta_{n}^{j} z+\left(\zeta_{n}^{j}\right)^{2} z^{2}+\cdots+\left(\zeta_{n}^{j}\right)^{n-1} z^{(n-1)}\right)^{h}\right|_{z^{n}=z} .
\end{aligned}
$$

The remainder of division of this polynomial by $(1-z)$ is equal to its value at the point $z=1$. Thus,

$$
\begin{aligned}
\left.\varphi_{n}\left(\left(1+z+z^{2}+\cdots+z^{n-1}\right)^{h}\right)\right|_{z=1} \\
=\frac{1}{n} \sum_{j=0}^{n-1}\left(1+\zeta_{n}^{j}+\left(\zeta_{n}^{j}\right)^{2}+\cdots+\left(\zeta_{n}^{j}\right)^{n-1}\right)^{h}=n^{h-1} .
\end{aligned}
$$

Obviously, $\alpha_{n, h-1}$ is the coefficient of $(1-z)$ in the Taylor series expansion for

$$
\varphi_{n}\left(\left(1+z+z^{2}+\cdots+z^{n-1}\right)^{h}\right)
$$

at the point $z=1$. Therefore,

$$
\alpha_{n, h-1}=-\lim _{z \rightarrow 1}\left(\varphi_{n}\left(\left(1+z+z^{2}+\cdots+z^{n-1}\right)^{h}\right)\right)^{\prime} .
$$

We have

$$
\begin{aligned}
\left(\frac { 1 } { n } \sum _ { j = 0 } ^ { n - 1 } \left(1+\zeta_{n}^{j} z+\right.\right. & \left.\left.\left(\zeta_{n}^{j}\right)^{2} z^{2}+\cdots+\left(\zeta_{n}^{j}\right)^{n-1} z^{(n-1)}\right)^{h}\right)^{\prime} \\
=\frac{h}{n} \sum_{j=0}^{n-1}(1+ & \left.\zeta_{n}^{j} z+\left(\zeta_{n}^{j}\right)^{2} z^{2}+\cdots+\left(\zeta_{n}^{j}\right)^{n-1} z^{(n-1)}\right)^{h-1} \times \\
& \times\left(\zeta_{n}^{j}+2\left(\zeta_{n}^{j}\right)^{2} z+\cdots+(n-1)\left(\zeta_{n}^{j}\right)^{n-1} z^{(n-2)}\right) .
\end{aligned}
$$


It now follows that

$$
\begin{gathered}
\lim _{z \rightarrow 1}\left(\frac{1}{n} \sum_{j=0}^{n-1}\left(1+\zeta_{n}^{j} z+\left(\zeta_{n}^{j}\right)^{2} z^{2}+\cdots+\left(\zeta_{n}^{j}\right)^{n-1} z^{(n-1)}\right)^{h}\right)^{\prime} \\
=\frac{h}{n} \sum_{j=0}^{n-1}\left(\sum_{k=0}^{n-1}\left(\zeta_{n}^{j}\right)^{k}\right)^{h-1}\left(\zeta_{n}^{j}+2\left(\zeta_{n}^{j}\right)^{2}+\cdots+(n-1)\left(\zeta_{n}^{j}\right)^{n-1}\right) \\
=\frac{h}{n} n^{h-1}(1+2+\ldots+(n-1))=\frac{1}{2} h(n-1) n^{h-1} .
\end{gathered}
$$

By using the relation

$$
\lim _{z \rightarrow 1}\left(\left.f\left(z^{n}\right)\right|_{z^{n}=z}\right)^{\prime}=\frac{1}{n} \lim _{z \rightarrow 1} f^{\prime}\left(z^{n}\right),
$$

we get

$$
\begin{aligned}
\alpha_{n, h-1} & =-\lim _{z \rightarrow 1}\left(\varphi_{n}\left(\left(1+z+z^{2}+\cdots+z^{n-1}\right)^{h}\right)\right)^{\prime} \\
& =-\frac{1}{n} \lim _{z \rightarrow 1}\left(\frac{1}{n} \sum_{j=0}^{n-1}\left(1+\zeta_{n}^{j} z+\left(\zeta_{n}^{j}\right)^{2} z^{2}+\cdots+\left(\zeta_{n}^{j}\right)^{n-1} z^{(n-1)}\right)^{h}\right)^{\prime} \\
& =-\frac{1}{2} h(n-1) n^{h-2} .
\end{aligned}
$$

Now we can compute $\operatorname{deg}\left(\mathcal{C}_{d}\right)$ and $\psi\left(\mathcal{C}_{d}\right)$.

\section{Theorem 2.4.}

$$
\begin{aligned}
\operatorname{deg}\left(\mathcal{C}_{d}\right) & =\lim _{z \rightarrow 1}(1-z)^{d} \mathcal{P}\left(\mathcal{C}_{d}, z\right) \\
& =\frac{1}{d !} \sum_{0 \leq j<d / 2}(-1)^{j}\left(\begin{array}{l}
d \\
j
\end{array}\right)\left(\frac{d}{2}-j\right)^{d-1}
\end{aligned}
$$

and

$$
\psi\left(\mathcal{C}_{d}\right)=\lim _{z \rightarrow 1}\left(-(1-z)^{d} \mathcal{P}\left(\mathcal{C}_{d}, z\right)\right)_{z}^{\prime}=\frac{1}{2} \operatorname{deg}\left(\mathcal{C}_{d}\right)
$$

Proof. Using Lemmas 1 and 2 we get

$$
\mathcal{P}\left(\mathcal{C}_{d}, z\right)=\sum_{0 \leq j<d / 2} \varphi_{d-2 j}\left(\frac{(-1)^{j} z^{j(j+1)}(1+z)}{\left(z^{2}, z^{2}\right)_{j}\left(z^{2}, z^{2}\right)_{d-j}}\right)
$$




$$
\begin{aligned}
= & \sum_{0 \leq j<d / 2} \varphi_{d-2 j}\left(\frac{(-1)^{j}}{2^{d-1} j !(d-j) !} \frac{1}{(1-z)^{d}}+\cdots\right) \\
= & \sum_{0 \leq j<d / 2} \frac{(-1)^{j}}{2^{d-1} j !(d-j) !} \varphi_{d-2 j}\left(\frac{1}{(1-z)^{d}}\right) \\
& +\sum_{0 \leq j<d / 2} \frac{(-1)^{j}(d+1)}{2^{d-1} j !(d-j) !}\left(\frac{1}{2} d-j-\frac{1}{2}\right) \\
\times & \left.\varphi_{d-2 j} \frac{1}{(1-z)^{d-1}}\right)+\cdots \\
= & \frac{1}{(1-z)^{d}} \sum_{0 \leq j<d / 2} \frac{(-1)^{j}(d-2 j)^{d-1}}{2^{d-1} j !(d-j) !} \\
& -\frac{1}{(1-z)^{d-1}} \frac{1}{2} \sum_{0 \leq j<d / 2} \frac{(-1)^{j}}{2^{d-1} j !(d-j) !} \\
& \times(d-2 j)^{d-2}(d-2 j-1)(d-1)+\frac{1}{(1-z)^{d-1}} \frac{1}{2} \sum_{0 \leq j<d / 2} \\
& \times \frac{(-1)^{j}}{2^{d-1} j !(d-j) !}(d+1)(d-2 j-1)(d-2 j)^{d-2}+\cdots \\
& \ldots
\end{aligned}
$$

Thus, the coefficient of $\frac{1}{(1-z)^{d}}$ is

$$
\begin{aligned}
\operatorname{deg}\left(\mathcal{C}_{d}\right) & =\sum_{0 \leq j<d / 2} \frac{(-1)^{j}(d-2 j)^{d-1}}{2^{d-1} j !(d-j) !} \\
& =\frac{1}{d !} \sum_{0 \leq j<d / 2}(-1)^{j}\left(\begin{array}{l}
d \\
j
\end{array}\right)\left(\frac{d}{2}-j\right)^{d-1}
\end{aligned}
$$

and the coefficient of $\frac{1}{(1-z)^{d-1}}$ is

$$
\psi\left(\mathcal{C}_{d}\right)=\frac{1}{2 d !} \sum_{0 \leq j<d / 2}(-1)^{j}\left(\begin{array}{l}
d \\
j
\end{array}\right)\left(\frac{d}{2}-j\right)^{d-1}
$$


3. Asymptotic behavior of $\operatorname{deg}\left(\mathcal{C}_{d}\right)$. Let us establish an integral representation for the $\operatorname{degree} \operatorname{deg}\left(\mathcal{C}_{d}\right)$. We denote by

$$
c_{d}:=\operatorname{deg}\left(\mathcal{C}_{d}\right) \cdot d !=\sum_{0 \leq j<d / 2}(-1)^{j}\left(\begin{array}{l}
d \\
j
\end{array}\right)\left(\frac{d}{2}-j\right)^{d-1} .
$$

The following statement holds:

\section{Lemma 3.1.}

(i) $c_{d}=2 \pi^{-1}(d-1) ! \int_{0}^{\infty} \frac{\sin ^{d} x}{x^{d}} d x$

(ii) $\operatorname{deg}\left(\mathcal{C}_{d}\right)>0$.

Proof.

(i) We have:

$$
\begin{aligned}
2 c_{d}= & \sum_{0 \leq j<d / 2}(-1)^{j}\left(\begin{array}{l}
d \\
j
\end{array}\right)\left(\frac{d}{2}-j\right)^{d-1} \\
& +\sum_{0 \leq j<d / 2}(-1)^{j}\left(\begin{array}{l}
d \\
j
\end{array}\right)\left(\frac{d}{2}-j\right)^{d-1} \\
= & \sum_{0 \leq j<d / 2}(-1)^{j}\left(\begin{array}{l}
d \\
j
\end{array}\right)\left(\frac{d}{2}-j\right)^{d-1} \\
& +\sum_{d / 2 \leq j<d}(-1)^{j}\left(\begin{array}{l}
d \\
j
\end{array}\right) \operatorname{sign}\left(\frac{d}{2}-j\right)\left(\frac{d}{2}-j\right)^{d-1} \\
= & \sum_{j=0}^{d}(-1)^{j}\left(\begin{array}{l}
d \\
j
\end{array}\right) \operatorname{sign}\left(\frac{d}{2}-j\right)\left(\frac{d}{2}-j\right)^{d-1} .
\end{aligned}
$$

We use that

$$
\frac{\pi}{2} \operatorname{sign}(a)=\int_{0}^{\infty} \frac{\sin a x}{x} d x
$$


Then

$$
\begin{aligned}
\pi c_{d} & =\frac{\pi}{2} \sum_{j=0}^{d}(-1)^{j}\left(\begin{array}{l}
d \\
j
\end{array}\right) \operatorname{sign}\left(\frac{d}{2}-j\right)\left(\frac{d}{2}-j\right)^{d-1} \\
& =\sum_{j=0}^{d}(-1)^{j}\left(\begin{array}{l}
d \\
j
\end{array}\right)\left(\frac{d}{2}-j\right)^{d-1} \int_{0}^{\infty} \frac{\sin (d / 2-j) x}{x} d x \\
& =\int_{0}^{\infty} \operatorname{Im}\left(\sum_{j=0}^{d}(-1)^{j}\left(\begin{array}{l}
d \\
j
\end{array}\right)\left(\frac{d}{2}-j\right)^{d-1} e^{i(d / 2-j) x}\right) \frac{d x}{x}, \quad i^{2}=-1 .
\end{aligned}
$$

This follows by the same method as in [7, Lemma 3.4.7]. We have:

$$
\begin{aligned}
\sin ^{d} \frac{x}{2} & =\left(\frac{e^{i x / 2}-e^{-i x / 2}}{2 i}\right)^{d} \\
& =\frac{1}{2^{d} i^{d}} \sum_{j=0}^{d}\left(\begin{array}{l}
d \\
j
\end{array}\right)\left(e^{i x / 2}\right)^{d-j}\left(e^{-i x / 2}\right)^{j} \\
& =\frac{1}{2^{d} i^{d}} \sum_{j=0}^{d}(-1)^{j}\left(\begin{array}{l}
d \\
j
\end{array}\right) e^{i x(d / 2-j)}
\end{aligned}
$$

Differentiating $d-1$ times with respect to $x$, we obtain

$$
\left(\sin ^{d} \frac{x}{2}\right)^{(d-1)}=\frac{i^{d-1}}{2^{d} i^{d}} \sum_{0 \leq j \leq d}(-1)^{j}\left(\begin{array}{l}
d \\
j
\end{array}\right)\left(\frac{d}{2}-j\right)^{d-1} e^{i x(d / 2-j)} .
$$

Hence,

$$
\operatorname{Im}\left(\sum_{j=0}^{d}(-1)^{j}\left(\begin{array}{l}
d \\
j
\end{array}\right)\left(\frac{d}{2}-j\right)^{d-1} e^{i(d / 2-j) x}\right)=2^{d}\left(\sin ^{d} \frac{x}{2}\right)^{d-1}
$$

Thus,

$$
c_{d}=\frac{1}{\pi} \int_{0}^{\infty} 2^{d}\left(\sin ^{d} \frac{x}{2}\right)^{d-1} \frac{d x}{x}=\frac{2}{\pi} \int_{0}^{\infty}\left(\sin ^{d} x\right)^{d-1} \frac{d x}{x} .
$$


Integrating by parts $d-1$ times, we obtain

$$
c_{d}=\frac{2(d-1) !}{\pi} \int_{0}^{\infty} \frac{\sin ^{d} x}{x^{d}} d x .
$$

(ii) It is enough to prove that

$$
\int_{0}^{\infty} \frac{\sin ^{d} x}{x^{d}} d x>0 .
$$

First of all, we prove that the integral is absolutely convergent. Let us split the integral into two parts:

$$
\int_{0}^{\infty} \frac{\sin ^{d} x}{x^{d}} d x=\int_{0}^{1} \frac{\sin ^{d} x}{x^{d}} d x+\int_{1}^{\infty} \frac{\sin ^{d} x}{x^{d}} d x
$$

Since $\lim _{x \rightarrow 0} \frac{\sin x}{x}=1$, the function $\left(\frac{\sin x}{x}\right)^{p}$ is continuous on $[0,1]$. Thus, the first integral is convergent. Since

$$
\left|\frac{\sin ^{d} x}{x^{d}}\right| \leq\left|\frac{1}{x^{d}}\right|
$$

then the second integral is absolutely convergent for $d>1$.

Now the integral can be represented in the form

$$
\begin{aligned}
& \int_{0}^{\infty} \frac{\sin ^{d} x}{x^{d}} d x \\
& =\sum_{j=0}^{\infty}\left(\int_{2 j \pi}^{(2 j+1) \pi} \frac{\sin ^{d} x}{x^{d}} d x+\int_{(2 j+1) \pi}^{4 j \pi} \frac{\sin ^{d} x}{x^{d}} d x\right) \\
& =\sum_{j=0}^{\infty}\left(\int_{2 j \pi}^{(2 j+1) \pi} \frac{\sin ^{d} x}{x^{d}} d x+\int_{2 j \pi}^{(2 j+1) \pi} \frac{\sin ^{d}(x+\pi)}{(x+\pi)^{d}} d x\right) \\
& \geq \sum_{j=0}^{\infty} \int_{2 j \pi}^{(2 j+1) \pi}\left(\frac{\sin ^{d} x}{x^{d}}-\frac{\sin ^{d} x}{(x+\pi)^{d}}\right) d x \\
& =\sum_{j=0}^{\infty} \int_{2 j \pi}^{(2 j+1) \pi} \frac{\sin ^{d} x}{x^{d}(x+\pi)^{d}}\left((x+\pi)^{d}-x^{d}\right) d x>0, \quad d>1 .
\end{aligned}
$$


For the case $d=1$, we have

$$
\int_{0}^{\infty} \frac{\sin x}{x} d x=\frac{\pi}{2}>0 .
$$

Condition $\operatorname{deg}\left(\mathcal{C}_{d}\right)>0$ is equivalent to the statement that the transcendence degree of the field of fractions of the algebra $\mathcal{C}_{d}$ is equal to $d$.

Interestingly, in the general case, the Wolstenholme formula holds:

$$
\int_{0}^{\infty} \frac{\sin ^{p} x}{x^{s}}=\frac{(-1)^{p-s / 2}}{(s-1) !} \frac{\pi}{2^{p}} \sum_{p-2 j>0}(-1)^{j}\left(\begin{array}{l}
p \\
j
\end{array}\right)(p-2 j)^{s-1},
$$

if $p-s$ is even, see [3, Problem 1033].

Finally, we deal with the asymptotic behavior of $\operatorname{deg}\left(\mathcal{C}_{d}\right)$ as $d$ tends to infinity. By the previous lemma, it is enough to determine the asymptotic behavior of

$$
\int_{0}^{\infty} \frac{\sin ^{d} x}{x^{d}} d x
$$

\section{Theorem 3.2.}

$$
\lim _{d \rightarrow \infty} d^{1 / 2} \int_{0}^{\infty} \frac{\sin ^{d} x}{x^{d}} d x=\frac{(6 \pi)^{1 / 2}}{2} .
$$

Proof. Write

$$
I=\lim _{d \rightarrow \infty} d^{1 / 2} \int_{0}^{\infty} \frac{\sin ^{d} x}{x^{d}} d x,
$$

and split the limit into two parts:

$$
\begin{aligned}
I= & \lim _{d \rightarrow \infty} d^{1 / 2} \int_{0}^{\infty} \frac{\sin ^{d} x}{x^{d}} d x \\
= & \lim _{d \rightarrow \infty} d^{1 / 2} \int_{0}^{\pi / 2} \frac{\sin ^{d} x}{x^{d}} d x \\
& +\lim _{d \rightarrow \infty} d^{1 / 2} \int_{\pi / 2}^{\infty} \frac{\sin ^{d} x}{x^{d}} d x .
\end{aligned}
$$


Since

$$
\begin{aligned}
\left|\int_{\pi / 2}^{\infty} \frac{\sin ^{d} x}{x^{d}} d x\right| & \leq \int_{\pi / 2}^{\infty} x^{-d} d x=\left.\lim _{b \rightarrow \infty} \frac{x^{-d+1}}{1-d}\right|_{\pi / 2} ^{b} \\
& =\frac{1}{(d-1) x^{d-1}} \longrightarrow 0,
\end{aligned}
$$

it follows that

$$
I=\lim _{d \rightarrow \infty} d^{1 / 2} \int_{0}^{\pi / 2} \frac{\sin ^{d} x}{x^{d}} d x .
$$

Fix $\varepsilon>0$ sufficiently small. Since $\sin x / x$ is monotonically decreasing as $0 \leq x \leq \pi / 2$, it follows that

$$
\frac{\sin x}{x} \leq \frac{\sin \varepsilon}{\varepsilon}=1-\frac{\varepsilon^{2}}{3 !}+\frac{\varepsilon^{4}}{5 !}-\cdots
$$

as $\varepsilon \leq x \leq \pi / 2$. It readily follows that there exists a strictly positive constant $a$ such that

$$
\int_{\varepsilon}^{\pi / 2} \frac{\sin ^{d} x}{x^{d}} d x=O\left(e^{-a \cdot d \varepsilon^{2}}\right) .
$$

For $0 \leq x \leq \varepsilon$, we have

$$
\left(\frac{\sin x}{x}\right)^{d}=\left(1-\frac{1}{6} x^{2}+O\left(\varepsilon^{4}\right)\right)^{d}=e^{-1 / 6 d x^{2}+O\left(d \varepsilon^{4}\right)} .
$$

Hence,

$$
\int_{0}^{\varepsilon} e^{-1 / 6 d x^{2}} d x=\frac{e^{O\left(d \varepsilon^{4}\right)}}{d^{1 / 2}} \int_{0}^{\varepsilon d^{1 / 2}} e^{-1 / 6 x^{2}} d x
$$

Now choose $\varepsilon=\ln d / \sqrt{d}$. Then the limit reduces to the Euler-Poisson integral:

$$
I=\lim _{d \rightarrow \infty} d^{1 / 2} \int_{0}^{\pi / 2} \frac{\sin ^{d} x}{x^{d}} d x=\sqrt{6} \int_{0}^{\infty} e^{-x^{2}} d x=\frac{\sqrt{6 \pi}}{2} .
$$

Thus, the asymptotic behavior of $\operatorname{deg}\left(\mathcal{C}_{d}\right)$ as $d \rightarrow \infty$ is as follows:

$$
\operatorname{deg}\left(\mathcal{C}_{d}\right)=\frac{c_{d}}{d !} \sim \sqrt{\frac{6}{\pi}} \frac{1}{d^{3 / 2}}
$$




\section{REFERENCES}

1. L. Bedratyuk, The Poincaré series for the algebra of covariants of a binary form, Int. J. Alg. 4 (2010), 1201-1207.

2. D. Benson, Polynomial invariants of finite groups, Lond. Math. Soc. Lect. Note 190, Cambridge University Press, Cambridge, 1993.

3. J. Edwards, A treatise on the integral calculus: With applications, examples, and problems, Macmillan and Co Limited, London, 1922.

4. D. Hilbert, Über die vollen Invariantsystemes, Math. Annal. 42 (1893), 313373.

5. V.L. Popov, Groups, generators, syzygies, and orbits in invariant theory, Transl. Math. Mono. 100, American Mathematical Society, Providence, RI, 1992.

6. T.A. Springer, Invariant theory, Lect. Notes Math. 585, Springer-Verlag, Berlin, 1977.

7.

8. R.P. Stanley, Invariants of finite groups and their applications to combinatorics, Bull. Amer. Math. Soc. 3 (1979), 475-511.

Khmelnitsky National University, Khmelnitsky, Instytutska, 11, 29016, UKRAINE

Email address: leonid.uk@gmail.com

Khmelnitsky National University, Khmelnitsky, Instytutska, 11, 29016, UKRAINE

Email address: ilashnadya@yandex.ua 